\title{
Relevance of anthropometric measurements as predictors of prevalent diabetes type II - A cross- sectional study on a Norwegian population
}

Anton Hasselgren ( $\nabla$ anton.karl.hasselgren@gmail.com )

Norges Teknisk-Naturvitenskapelige Universitet Fakultet for Medisin og Helsevitenskap https://orcid.org/0000-0002-6245-6041

Biraj M Karmacharya

Kathmandu University School of Medical Sciences

Ann-Katrin Stensdotter

Norges Teknisk-Naturvitenskapelige Universitet Fakultet for Medisin og Helsevitenskap

Research article

Keywords: Diabetes mellitus type 2, Anthropometric indices, HUNT, Optimal cut-offs.

Posted Date: March 24th, 2020

DOI: https://doi.org/10.21203/rs.2.22518/v2

License: (c) (i) This work is licensed under a Creative Commons Attribution 4.0 International License. Read Full License 


\section{Abstract}

Background The association of overweight and diabetes mellitus type 2 varies with geographical area and ethnicity, and there is incoherent evidence as to which anthropometric index best predicts prevalent diabetes mellitus type 2 in different populations. The rationale for this study was to contribute to the understanding of how anthropometric indices perform as a screening tool for diabetes mellitus type 2 . Hence, the objective was to determine the predictive potential of anthropometric indices to screen prevalent diabetes mellitus type 2 in the Norwegian population.Methods This study used cross-sectional data from the Health Study in Nord-Trøndelag (HUNT) to determine the potential association of waist-toheight ratio, waist-to-hip ratio, waist-circumference and body mass index with prevalent diabetes mellitus type 2 through logistic regression analysis. Receiver Operating Characteristic (ROC) curves were used to determinate the predictive potential of the anthropometric indices. Youden's index was applied to determinate the optimal cut-off points for each anthropometric index.Results The results suggest that the anthropometric indices performed differently within the Norwegian population with waist-to-hip ratio and waist-to-height ratio being the strongest predictors with (ROC) of $0.746(0.735-0.757)$ and $0.741(0.730$ 0.752). The predictive potential for the investigated anthropometric indices was generally stronger for women than men.Interpretation When using anthropometric indices to screen prevalent diabetes mellitus type 2 , the choice of anthropometric index and cut-off points should be considered based on country and the ethnic characteristics of the population.

\section{Background}

It is estimated that 415 million people in world suffer from some form of diabetes. Of those $46.5 \%$ are undiagnosed. By 2040 the International Diabetes Federation (IDF) predicts that 642 million people will have diabetes type 2 (DMT2) [1]. It is also estimated that 318 million adults have impaired glucose tolerance, which is a form of pre-diabetes. Estimates from WHO tells us that high blood glucose counts as the third highest risk factor for premature mortality [2]. Over 2.2 million deaths were caused by high blood sugar in 2012 and 1.5 million deaths were directly caused by diabetes during the same year [3]. Diabetes Mellitus type 2 counts for $90-95 \%$ of all diabetes cases worldwide [4].

According to the diabetes atlas $7^{\text {th }}$ edition [1], approximately $6 \%$ of the 5.1 million people that populates Norway had diabetes in 2015 [5]. About $90 \%$ of all diabetes cases were DMT2. The projections for year 2040 do not differ from 2015; the prevalence of DMT2 is expected to stay the same.

It is well-known that anthropometric measures such as body mass index (BMI), waist-circumference (WC), waist-to-height ratio (WHtR) and waist-to-hip ratio (WHR) are associated with prevalence of diabetes DMT2 [6-8]. Even those who are not classified as obese or overweight by standard screening methods (i.e., BMI) may still have a higher ratio of body fat and this may increase the risk of DMT2, especially if that extra fat is distributed around the abdominal area [9]. However, the association appears to vary between ethnicities [6-8]. Indicators of overweight and central obesity are therefore important factors to 
be able to early identify those individuals with prevalent DMT2. Such indicators are also often incorporated in general guidelines for early detection of diabetes [10].

Several of studies have indicated that, both WC and WHtR seem to perform better as a predictor of DMT2 compared to BMI, with Receiver Operating Characteristic (ROC) values. The review included studies conducted on men and women with an ethnicity of Caucasian-white, Asian and Central American [11].

A study from 2010 suggested that there is no anthropometric measure that could perform across ethnicities; the authors found a large variation on the predictive potential of anthropometric measures with DMT2, based on ethnicity and sex [12].

To our knowledge, there have been no studies that investigate the association between DMT2 and different anthropometric indices in Norway using a larger, population-based data sample. This study investigated the prevalence of DMT2 in a Norwegian population with the objective to determine the predictive potential of anthropometric indices to screen prevalent DMT2 in this population: First, we determined the association of WHtR, WHR, WC and BMI with prevalent DMT2, second, we estimated the optimal cut-off points for the same factors to predict prevalent DMT2. This may be of clinical value by contributing to the understanding of which anthropometric measurement performs best in terms of predicting prevalent DMT2 in a Norwegian population.

\section{Method}

\subsection{Data collection}

Data from 50042 who participated in the third wave of the Norwegian population-based cohort study: The Nord-Trøndelag health study (HUNT). There have been three waves of data collection so far: HUNT1 (1984-1986), HUNT2 (1995-1997) and HUNT3 (2006-2008), with a fourth currently ongoing (2018). This study used the cross-sectional data from the most recent wave of data collection, HUNT3. The population in the county of Nord-Trøndelag is relatively ethnical homogenous, with only $3 \%$ nonCaucasians. It is also considered to be fairly representative for the whole population of Norway in terms of geographical, demographic and employment structures [14]. An invitation with the health questionnaire was sent by mail to all citizens in the county of Nord-Trøndelag who were aged $\geqslant 20$ years. Details concerning the questionnaires and examinations have been described elsewhere [14].

The ones who chose to participate completed an extensive questionnaire (including information on previously known diabetes), clinical examination and blood sampling [14]. Weight and height were measured with the participants wearing light clothes and without shoes. WC was measured horizontally at the level of the umbilicus with a non-elastic tape-measure when the participants was standing with the arms relaxed. $\mathrm{HC}$ was measured around the buttocks, below the iliac crest were the circumference appeared to be largest [14].

\subsection{Sample}


All data used in this study were de-identified and the authors had no access to any identifier key. This study included all those who were non-pregnant and had complete data $(N=50,042,98.5 \%$ of the participants, Figure 1) on the variables listed in Table 1.

Table 1 - Variables included in the analysis

\begin{tabular}{|l|l|}
\hline Dependent Variables & Definition \\
\hline DMT2 & Stated that diagnoses of DMT2 have been made by a medical doctor* \\
\hline Independent Variables & Age at the time of the data collection \\
\hline Age & Male or female \\
\hline Sex & Smoking at least one cigarette per day \\
\hline Smoking & Kilograms/height in meters ${ }^{2}$ \\
\hline BMI & Waist circumference in centimeters \\
\hline WC & Hip circumference in centimeters \\
\hline HC & Waist circumference/Hip circumference \\
\hline WHR & Waist circumference/Height in centimeters \\
\hline WHtR & Weight in kilograms \\
\hline Weight & Height in centimeters \\
\hline Height & $\begin{array}{l}\text { those who stated that they had diabetes type 1, Latent Autoimmune Diabetes of Adults (LADA), Maturity Onset } \\
\text { Diabetes in Young (MODY) or unspecified diabetes were not categorized as type 2 diabetics. }\end{array}$ \\
\hline
\end{tabular}

A previous study has validated the self-reported diabetes question in HUNT by comparison with medical records. The result showed that the answers were verified in $96 \%$ of the cases [15]. The cut-off levels used are those recommended by the WHO and the International Diabetes Federation (IDF) [16].

\subsection{Statistical analysis}

Normal distribution was assessed and verified using histograms and Q-Q plots. Descriptive statistics of all variables are presented as numerical data. Two-sample t-test was used to show any potential group differences in the data between men and women and between diabetics and non-diabetics. For the categorical variables, a $\chi 2$ test was used to compare any potential differences between the groups. All categorical data were expressed as percentages. BMI was presented as a mean and categorized by general overweight cut-offs according to WHO [17]. To consider confounding factors for odds ratio; 3 logistic regression models were developed and applied to determine the association between DMT2 and the anthropometric measures. In the first model, no variables were adjusted for. The second model adjusted for sex. The third model adjusted for sex, age, and smoking (yes or no). Smoking have shown to have a clear association with diabetes type 2 [23]. The categorical anthropometric measures used were BMI, WC, WHR, and WHtR included as independent variables in all three models. Before running the logistic regression models, the continuous anthropometric indices BMI, WC, WHR and WHtR were 
categorized into four levels using the quartiles of P25, P50 and P75 as cut-off values with the lowest quartiles as a reference.

To determine the predictive potential of the anthropometric measures for DMT2, the ROC curves, area under the curve (AUCs) and their 95\% Cl were calculated for BMI, WC, WHR or WHtR. Since ROC are unadjusted, these are presented according to sex. A p-value of $<0.05$ was considered statistically significant in the analysis.

The optimal cut-off values were calculated using Youden's index for the anthropometric indices (sensitivity + specificity - 1) [18]. The index was defined for all points of the ROC curve. The maximum value of the index defined the optimal cut-off point for predicting prevalent DMT2.

Statistical analysis was performed using SPSS version 25 (IBM, Ehningen, Germany).

\section{Result}

\subsection{Descriptive}

A summary of the characteristics for the participants is shown in Table 2. Slightly more women (54.4\%) than men were included in the sample. The data shows that prevalence of DMT2 was higher among men (4.3\%) compared to women (3.3\%). The total prevalence for the sample was 3.7\%. Men had a significantly higher average age, weight, height, BMI, HC, WC, WHR, and WHtR compared to women. Almost twice as many men (22.2\%) were smokers compared to women (11.7\%), with a total smoking prevalence of $16.5 \%$.

Table 2 - Descriptive statistics according to sex 


\begin{tabular}{|c|c|c|c|c|}
\hline Characteristics & Men $(n=22,830)$ & Women $(n=27,212)$ & Total $(n=50,042)$ & P-value \\
\hline DMT2, n (\%) & $975(4.3)$ & $901(3.3)$ & $1,876(3.7)$ & $<0.001$ \\
\hline Smoking, n (\%) & $5,072(22.2)$ & $3,195(11.7)$ & $8,267(16.5)$ & $<0.001$ \\
\hline Age (years) & $53.6 \pm 15.6$ & $52.8 \pm 16.3$ & $53.1 \pm 16.1$ & $<0.001$ \\
\hline Weight (kg) & $86.9 \pm 13.3$ & $72.9 \pm 13.7$ & $79.2 \pm 15.2$ & $<0.001$ \\
\hline Height (cm) & $177.8 \pm 6.7$ & $164.6 \pm 6.4$ & $170.6 \pm 9.3$ & $<0.001$ \\
\hline $\mathrm{HC}(\mathrm{cm})$ & $103.6 \pm 6.6$ & $103.8 \pm 9.3$ & $103.7 \pm 8.2$ & 0.024 \\
\hline $\mathrm{WC}(\mathrm{cm})$ & $97.4 \pm 10.5$ & $90.3 \pm 12.8$ & $93.6 \pm 12.3$ & $<0.001$ \\
\hline BMI $\left(\mathrm{kg} / \mathrm{m}^{2}\right)$ & $27.5 \pm 3.8$ & $26.9 \pm 4.9$ & $27.2 \pm 4.4$ & $<0.001$ \\
\hline$<18.49, \mathrm{n}(\%)$ & $74(0.3)$ & $238(0.9)$ & $312(0.6)$ & \\
\hline $18.5-24.99, \mathrm{n}(\%)$ & $5,678(25)$ & $10,414(45.9)$ & $16,092(32)$ & \\
\hline $25-29.99, \mathrm{n}(\%)$ & $11,955(52.4)$ & $10,346(37.9)$ & $22,301(44.4)$ & \\
\hline$>30, \mathrm{n}(\%)$ & $5,123(22.4)$ & $6,398(22.3)$ & $11,521(22.9)$ & \\
\hline WHR & $0.94 \pm 0.07$ & $0.87 \pm 0.07$ & $0.90 \pm 0.08$ & $<0.001$ \\
\hline WHtR & $0.55 \pm 0.06$ & $0.55 \pm 0.08$ & $0.55 \pm 0.07$ & 0.04 \\
\hline
\end{tabular}

Results are shown as means $\pm S D$ unless stated otherwise.

The comparison of characteristics between men and women (unpaired Student's t-test for numerical data, $\chi^{2}$ test for categorical data).

BMI, body mass index; DMT2, diabetes mellitus type 2; HC, hip circumference; WC, waist circumference; WHR, waistto-hip ratio; WHtR, waist-to-height ratio.

Table 3 shows descriptive statistics of the diabetic and non-diabetic group (diabetic referring to DMT2). Average age, weight, $\mathrm{HC}, \mathrm{WC}, \mathrm{BMI}, \mathrm{WHR}$, and WHtR were all significantly higher in the diabetic group. Of the total sample, $67.3 \%$ were either overweight or obese according to universal cut-offs for $\mathrm{BMI}$ and $87.3 \%$ were either obese or overweight in the diabetic group. The group without diabetes had a higher prevalence of smoking (16.6\%) compared to the diabetic group (13.8\%).

Table 3 - Descriptive statistics according to diabetic. and non-diabetic groups 


\begin{tabular}{|c|c|c|c|c|}
\hline Characteristics & Diabetic $(n=1,876)$ & Non-diabetic $(n=48,166)$ & $\begin{array}{l}\text { Total } \\
(n=50,042)\end{array}$ & P-value \\
\hline Smoking, n (\%) & $260(13.8)$ & $8,007(16.6)$ & $8,267(16.5)$ & $<0.001$ \\
\hline Age (years) & $65.4 \pm 11.8$ & $52.7 \pm 15$ & $53.1 \pm 16.1$ & $<0.001$ \\
\hline Weight (kg) & $86.5 \pm 16.6$ & $78 \pm 15.1$ & $79.2 \pm 15.2$ & $<0.001$ \\
\hline Height $(\mathrm{cm})$ & $169 \pm 9.4$ & $170.6 \pm 9.2$ & $170.6 \pm 9.3$ & $<0.001$ \\
\hline $\mathrm{HC}(\mathrm{cm})$ & $106.7 \pm 9.3$ & $103.6 \pm 8.1$ & $103.7 \pm 8.2$ & $<0.001$ \\
\hline $\mathrm{WC}(\mathrm{cm})$ & $103.4 \pm 12.6$ & $93.2 \pm 12.1$ & $93.6 \pm 12.3$ & $<0.001$ \\
\hline BMI $\left(\mathrm{kg} / \mathrm{m}^{2}\right)$ & $30.2 \pm 5.0$ & $27.1 \pm 4.3$ & $27.2 \pm 4.4$ & $<0.001$ \\
\hline$<18.49, \mathrm{n}(\%)$ & $4(0.2)$ & $308(0.6)$ & $312(0.6)$ & \\
\hline $18.5-24.99, \mathrm{n}(\%)$ & $235(12.5)$ & $15,857(32.8)$ & $16,092(32)$ & \\
\hline 25-29.99, n (\%) & $750(40)$ & $21,551(44.6)$ & $22,301(44.4)$ & \\
\hline$>30, \mathrm{n}(\%)$ & $887(47.3)$ & $10,634(22)$ & $11,521(22.9)$ & \\
\hline WHR & $0.97 \pm 0.07$ & $0.9 \pm 0.08$ & $0.90 \pm 0.08$ & $<0.001$ \\
\hline WHtR & $0.61 \pm 0.08$ & $0.55 \pm 0.07$ & $0.55 \pm 0.07$ & $<0.001$ \\
\hline
\end{tabular}

Results are shown as means $\pm S D$ unless stated otherwise.

The comparison of characteristics between diabetic and non-diabetic (unpaired Student's t-test for numerical data, $\chi^{2}$ test for categorical data).

BMI, body mass index; HC, hip circumference; WC, waist circumference; WHR, waist-to-hip ratio; WHtR, waist-toheight ratio.

\subsection{Associations between DMT2 and anthropometric measures}

In the regression analysis, the lowest quartile was taken as the reference in the analysis. In addition to unadjusted analysis, two models where applied. The first one adjusting only for sex and the second one for sex, age and smoking. Each of the four anthropometric indices showed a statistically significant increase in OR when comparing the quartiles to reference values, with the largest difference found in the highest quartiles in all models. The increase in OR across the quartiles indicates that the likelihood of having diabetes type 2 increases for higher values of all the four anthropometric indices. In the unadjusted model, OR was largest for WHtR: 12.34 (9.98 - 15.24) followed by the highest quartile in WHR: 11.16 (9.14-13.63), when comparing confidents intervals. The adjusted models showed a statistically significant difference in OR compared to the unadjusted model only in three cases: The OR for the highest quartile for WHR in model 1, 14.64 (11.89-18.02) was larger than in Model 2, 8.92 (7.2211.02). For WHtR, the OR for the second to highest quartile was larger in model 2, 3.22 (2.56-4.03) compared to the unadjusted OR, 5.22 (4.18-6.51) in the same quartile. Lastly, the highest quartile for WHtR had a lower OR in model 2 compared to model 1 and the unadjusted OR, in the same quartile (Table 4).

Table 4 - OR and 95\% CI of the presence of DMT2 according to quartiles of anthropometric index 


\begin{tabular}{|c|c|c|c|c|c|c|c|c|}
\hline Variable & $\mathbf{N}$ & $\%$ & Unadjusted & & Model 1* & & Model 2** & \\
\hline BMI & & & Odds Ratio & $95 \% \mathrm{CI}$ & Odds Ratio & $95 \% \mathrm{CI}$ & Odds Ratio & $95 \% \mathrm{CI}$ \\
\hline$<24.1$ & 12,235 & $24.5 \%$ & reference & & reference & & reference & \\
\hline $24.1-26.6$ & 12,597 & $25.2 \%$ & 1.83 & $1.51-2.23$ & 1.76 & $1.45-2.15$ & 1.53 & $1.25-1.86$ \\
\hline $26.7-29.5$ & 12,455 & $24.9 \%$ & 3.10 & $2.58-3.72$ & 2.95 & $2.46-3.55$ & 2.45 & $2.03-2.94$ \\
\hline $29.6+$ & 12,755 & $25.5 \%$ & 6.23 & $5.25-7.39$ & 6.06 & $5.10-7.19$ & 5.18 & $4.35-6.16$ \\
\hline \multicolumn{9}{|l|}{ WC } \\
\hline$<85$ & 11,752 & $23.5 \%$ & reference & & reference & & reference & \\
\hline $85-93$ & 11,866 & $23.8 \%$ & 2.01 & $1.61-2.51$ & 2.06 & $1.65-2.57$ & 1.67 & $1.33-2.08$ \\
\hline $93-101$ & 12,918 & $25.8 \%$ & 3.38 & $2.75-4.14$ & 3.5 & $2.84-4.30$ & 2.53 & $2.08-3.13$ \\
\hline $101.1+$ & 13,476 & $26.9 \%$ & 8.57 & $7.08-10.37$ & 8.91 & $7.33-10.82$ & 6.19 & $5.08-7.54$ \\
\hline \multicolumn{9}{|l|}{ WHR } \\
\hline$<.85$ & 12,533 & $25.0 \%$ & reference & & reference & & reference & \\
\hline $0.85-0.89$ & 12,630 & $25.2 \%$ & 2.15 & $1.71-2.71$ & 2.38 & $1.89-2.99$ & 1.94 & $1.53-2.44$ \\
\hline $0.90-0.94$ & 12,316 & $24.6 \%$ & 4.27 & $3.45-5.29$ & 5.16 & $4.15-6.40$ & 3.66 & $2.94-4.55$ \\
\hline $0.95+$ & 12,563 & $25.1 \%$ & 11.16 & $9.14-13.63$ & 14.64 & $11.89-18.02$ & 8.92 & $7.22-11.02$ \\
\hline \multicolumn{9}{|l|}{ WHtR } \\
\hline$<0.50$ & 12,603 & $25.2 \%$ & reference & & reference & & reference & \\
\hline $0.50-0.53$ & 12,520 & $25.0 \%$ & 2.57 & $2.02-3.27$ & 2.45 & $1.93-3.11$ & 1.85 & $1.46-2.36$ \\
\hline $0.54-0.58$ & 12,510 & $25.0 \%$ & 5.22 & $4.18-6.51$ & 4.96 & $3.97-6.19$ & 3.22 & $2.56-4.03$ \\
\hline $0.59+$ & 12,429 & $24.8 \%$ & 12.34 & $9.98-15.24$ & 12.30 & $9.96-15.2$ & 7.19 & $5.80-8.92$ \\
\hline
\end{tabular}

Dependent variable: DMT2

*adjusted for sex

**adjusted for sex, age and smoking

BMI, body mass index $\left(\mathrm{kg} / \mathrm{m}^{2}\right)$; CI, confidence interval; DMT2, diabetes mellitus type 2; OR, odds ratio; WC, waist circumference (centimeters); WHR, waist-to-hip ratio; WHtR, waist-to-height ratio.

\subsection{Predictable values}

WHR was the strongest predictor for DMT2 followed by WHtR, WC, and BMI. The predictable value for BMI was significantly weaker than for all the other indices. The predictable value for WC was significantly weaker compared only to WHtR. The order of indices did not differ between men and women but the predictable potential was statistically higher for women than men according to the AUC. Table 5 shows the area under the ROC-curve, stratified for sex.

Cut-offs according to Yoden's index indicate a trend toward lower cut-offs for women than men, for WHR and WC. Other indices show small differences between groups (Table 6).

Table 6 - Optimal cut-off points of BMI, WHtR, WHR and WC according to Youden's index

\begin{tabular}{llll} 
Optimal cut-offs* & Total & Men & Women \\
\hline BMI & 28.35 & 28.25 & 28.35 \\
\hline WHtR & 0.57 & 0.58 & 0.57 \\
\hline WHR & 0.93 & 0.96 & 0.90 \\
WC & 97.25 & 98.70 & 91.00
\end{tabular}

*According to Youden's index 
Table 5 - Area under the curve with 95\% CI and Optimal cut-off points

Area Under the Curve (men)

\begin{tabular}{llll}
\hline Test Result Variables & AUC & $\mathbf{9 5 \%}$ CI \\
BMI & 0.666 & 0.648 & -0.684 \\
WHtR & 0.723 & 0.707 & -0.739 \\
WHR & 0.734 & 0.718 & -0.750 \\
WC & 0.698 & 0.681 & -0.715 \\
\hline \multicolumn{4}{l}{} \\
\multicolumn{4}{l}{ Area Under the Curve (women) } \\
Test Result Variables & AUC & $\mathbf{9 5 \%}$ CI \\
BMI & 0.713 & 0.696 & -0.729 \\
WHtR & 0.764 & 0.749 & -0.779 \\
WHR & 0.770 & 0.755 & -0.786 \\
WC & 0.742 & 0.726 & -0.757
\end{tabular}

BMI, body mass index $\left(\mathrm{kg} / \mathrm{m}^{2}\right) ; \mathrm{WC}$, waist circumference (centimeters); WHR, waist-tohip ratio; WHtR, waist-to-height ratio.

\section{Discussion}

WHR had the strongest association with DMT2, followed by WHtR, WC and BMI. The predictable values differed between men and women, showing lower cut-offs for women for WHR and WC. The potential confounders adjusted for in the regression models (sex, age and smoking) did not to

BMI, body mass index $\left(\mathrm{kg} / \mathrm{m}^{2}\right) ; W C$, waist circumference (centimeters); WHR, waist-to-hip ratio; WHtR, waist-toheight ratio; AUC, area under the curve; CI, confidence interval

affect the OR statistically except in a few cases. Notably, OR in the unadjusted model was highest for WHtR, but in the adjusted model higher for WHR, which in total placed WHR as the strongest predictor for DMT2.

While the cross-sectional design of this study does not make it possible to draw any conclusions about causality, it still provides valuable insight in the association between anthropometric indices and DMT2. The result suggests that we need to be aware of the different predictive potentials of anthropometric indices for screening prevalent DMT2.

This result is in line with what a previous research have concluded [20]. The prevalence of DMT2 in the sample (3.9\%) is slightly lower than figures mentioned in the current literature [19]. Selection bias could however explain this difference. A high proportion of individuals were categorized either overweight or obese in the sample (67.3\%) with and an average BMI of 27.2 .

The AUC suggests that the predictive potential of WHR is highest in this sample, followed by WHtR, WC and BMI. The order is the same for both men and women. This result is in line with data from systematic reviews, even though AUC values were higher in this study than previously reported in other studies [11, 20]. All the tested anthropometric indices worked statistically significantly better for women compared to men. Whether this could be explained by the difference in anthropometry between men and women or by a potential bias is not clear. A systematic review proposed that the following anthropometric cut-offs should apply for a Caucasian-white population: WC men; 97-99 cm, WC women; 85 cm, WHR men; 0.95 and WHR women; 0.83-0.85 [13]. The optimal cut-off values presented in our research are similar to what previous studies have reported, for example in this study the best cut-off points for Europeans were suggested to be: BMI 28.2, WC $103.4 \mathrm{~cm}$ and WHtR 0.607 for men. For women the suggestion was: BMI 26.7, WC $91.4 \mathrm{~cm}$ and WHtR 0.584 [22] 
However, the present result indicates that for Norwegian women, the cut-offs for WHR and BMI should be higher than what other studies have suggested for European populations [13]. This could possibly be explained by the fact that Norwegian women are in average taller than central and south Europeans [21]. The present result indicates that the difference between men and women could be small for BMI and WHtR, but that men should have a higher cut-off point than women for WC and WHR. This is in line with the current litterateur even though the difference in WHR seem to be smaller for the Norwegian population than previously suggested for a general Caucasian-white population [22].

The suggested optimal cut-off estimates found based on this large population based sample, should be taken into considiration when revising national guidelines for Norway respectivly, and caution should be exercised when applying universal cut-offs for WC and BMI since these differ from the findings in this country specific sample.

To our knowledge, there are no studies that have investigated the predictive potential of anthropometric indices for DMT2 on a large population-based sample in Norway, and there is currently incoherent evidence regarding the association of anthropometric indices and prevalent DMT2 presented in the literature. One strength of this study is the large population-based sample with standardized measurements that increase the generalizability of the results.

In the regression model, potential confounders from the available variables were applied (sex, age, and smoking). The regression analysis showed that the covariates did not have a statistical impact on the association in for the majority of cases, therefore they were not included in the ROC curve analysis which were only stratified for sex. This study did not include confounders like physical activity and diet due to low reliability when such data is self-reported.

\subsection{Conclusions}

Our study confirms that WHtR, WHR, WC and BMI perform differently as predictive tools for prevalent DMT2 in the Norwegian population. The result contributes to clarify which overweight index are strongest associated with prevalent DMT2 and suggest that WHR may provide the best index to detect DMT2 in Norway. This study also indicates that anthropometric indices detect DMT2 better in Norwegian women than men. The gender difference should be considered when screening for DMT2 in a Norwegian population. We have concluded that the currently mostly used anthropometric index, BMI has not the strongest association with DMT2 compared to WHR, WC and WHtR which is important to consider when designing screening programs for DMT2 in a Norwegian population.

\section{Abbreviations}

AUC Area Under the Receiver Operating Characteristic Curve

BMI Body Mass Index

$\mathrm{Cl} \quad$ Confidence Interval 


\begin{tabular}{ll} 
CVD & Cardio Vascular Disease \\
DMT2 & Diabetes Mellitus Type 2 \\
FBG & Fasting Blood Glucose \\
HC & Hip Circumference \\
HUNT & The Nord-Trøndelag Health Study \\
IDF & International Diabetes Federation \\
LADA & Latent Autoimmune Diabetes of Adults \\
mg/dl & Milligram per Deciliter \\
mmol/L & Millimole per Liter \\
MODY & Maturity Onset Diabetes in Young \\
NCD & Non-Communicable Disease \\
OR & Odds Ratio \\
ROC & Receiver Operating Characteristic \\
WC & Waist Circumference \\
WHO & World Health Organization \\
WHR & Waist to Hip Ratio \\
WHtR & Waist to Height Ratio \\
\hline
\end{tabular}

\section{Declarations}

\section{Ethics approval and consent to participate}

This research project was approved by the Norwegian Ethical Committee (2017/2047/REK midt). nformed consent was given from all the participants at the time of the data collection. All data used in this study were de-identified and the authors had no access to any identifier key. The consent also include consent for publication

\section{Consent to publication}

Not applicable 


\section{Availability of data and material}

The data used in this study is owned by the research team behind the HUNT and is not open access. An overview of data can be found here: https://hunt-db.medisin.ntnu.no/hunt-db/\#/survey/NT3

\section{Competing interests}

None of the authors have competing interests.

\section{Funding}

No external funding were given to this research.

\section{Authors contribution}

All authors have made a substantial contribution to the paper. $\mathrm{AH}, \mathrm{BK}$ and $\mathrm{AS}$ have all contributed in the design of the study. $\mathrm{AH}$ and $\mathrm{BK}$ have contributed in the data analyses and $\mathrm{AH}$ and $\mathrm{AS}$ have contributed in the methodology and discussion of the results. All authors have revised and approved the final version of the manuscript.

\section{Acknowledgment}

No other acknowledgment.

\section{References}

1. Federation, I.D., F Diabetes Atlas update poster. 7th edition. 2014, International Diabetes Federation: Brussels, Belgium.

2. World Health Organization. Diabetes Country Profiles 2016. 2016 [cited 2018 01.05]; Available from: http://www.who.int/diabetes/country-profiles/npl_en.pdf?ua=1.

3. World Health Organization, Global report on diabetes. 2016: Geneva.

4. Guariguata, L., et al., Global estimates of diabetes prevalence for 2013 and projections for 2035. Diabetes Res Clin Pract, 2014. 103(2): p. 137-49.

5. OECD. Country statistical profile: Norway. 2018 [cited 2018 01.05]; Available from: http://dx.doi.org/10.1787/csp-nor-table-2018-1-en.

6. McKeigue, P.M., B. Shah, and M.G. Marmot, Relation of central obesity and insulin resistance with high diabetes prevalence and cardiovascular risk in South Asians. Lancet, 1991. 337(8738): p. 382-6.

7. James, W.P.T., et al., Overweight and obesity (high body mass index). Comparative quantification of health risks: global and regional burden of disease attributable to selected major risk factors, 2004. 1: p. 497-596.

8. Whincup, P.H., et al., Early evidence of ethnic differences in cardiovascular risk: cross sectional comparison of British South Asian and white children. Bmj, 2002. 324(7338): p. 635. 
9. Diagnosis and classification of diabetes mellitus. Diabetes Care, 2014. 37 Suppl 1: p. S81-90.

10. Griffin, S.J., et al., Diabetes risk score: towards earlier detection of type 2 diabetes in general practice. Diabetes Metab Res Rev, 2000. 16(3): p. 164-71.

11. Ashwell M, Hsieh SD. Six reasons why the waist-to-height ratio is a rapid and effective global indicator for health risks of obesity and how its use could simplify the international public health message on obesity. International journal of food sciences and nutrition. 2005 Jan 1;56(5):303-7.

12. Qiao, Q. and R. Nyamdorj, Is the association of type II diabetes with waist circumference or waist-tohip ratio stronger than that with body mass index? Eur J Clin Nutr, 2010. 64(1): p. 30-4.

13. Qiao, Q. and R. Nyamdorj, The optimal cutoff values and their performance of waist circumference and waist-to-hip ratio for diagnosing type I/ diabetes. Eur J Clin Nutr, 2010. 64(1): p. 23-9.

14. Jostein Holmen, K.M., Øystein Krüger, Arnulf Langhammer, Turid Lingaas Holmen, Grete H. Bratberg, Lars Vatten and Per G. Lund-Larsen, The Nord-Trøndelag Health Study 1995-97 (HUNT 2): Objectives, contents, methods and participation. Norsk Epidemiologi, 2003. 13(1): p. 19-32.

15. Midthjell, K., et al., Is questionnaire information valid in the study of a chronic disease such as diabetes? The Nord-Trøndelag diabetes study. Journal of Epidemiology \& Community Health, 1992. 46(5): p. 537-542.

16. World Health Organization, Definition and diagnosis of diabetes mellitus and intermediate hyperglycaemia: report of a WHO. 2006.

17. World Health Organization. Global database on body mass index. 2006 [cited 2018 01.05]; Available from: http://www.who.int/bmi/index.jsp.

18. Fluss, R., D. Faraggi, and B. Reiser, Estimation of the Youden Index and its associated cutoff point. Biometrical journal, 2005. 47(4): p. 458-472.

19. Lars Christian Stene, H.S.a.H.L.G., Diabetes in Norway. 2017, Norwegian institute of public health.

20. Ashwell, M., P. Gunn, and S. Gibson, Waist-to-height ratio is a better screening tool than waist circumference and BMI for adult cardiometabolic risk factors: systematic review and meta-analysis. Obes Rev, 2012. 13(3): p. 275-86.

21. Cavelaars, A., et al., Persistent variations in average height between countries and between socioeconomic groups: an overview of 10 European countries. Annals of human biology, 2000. 27(4): p. 407-421.

22. A., D.V., et al., How does ethnicity affect the association between obesity and diabetes? Diabetic Medicine, 2007. 24(11): p. 1199-1204.

23. Willi C, Bodenmann P, Ghali WA, Faris PD, Cornuz J. Active smoking and the risk of type 2 diabetes: a systematic review and meta-analysis. Jama. 2007 Dec 12;298(22):2654-64

\section{Figures}




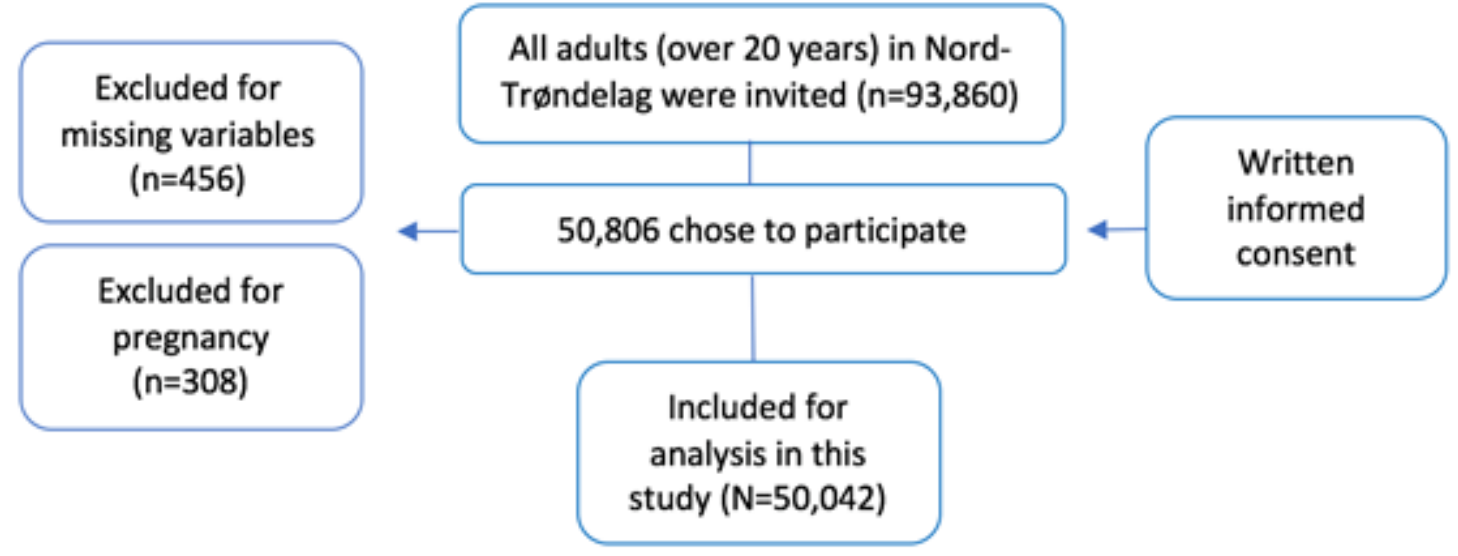

Figure 1

Data collection flow chart 